\title{
Modelo estratégico propuesto para transferencia de conocimiento en apoyo a la toma de decisiones en Marketing.
}

\author{
Mario Barcelo-Valenzuela, Fernando Bojórquez-Castillón, Federico Cirett Galán \\ Universidad de Sonora, Departamento de Ingeniería Industrial, Rosales y Blvd. Luis Encinas S/N CP. 83000, Hermosillo, \\ Sonora, México. \\ mbarcelo@industrial.uson.mx, \\ fbojorquezcastillon@gmail.com \\ fcirett@gmail.com
}

\begin{abstract}
Resumen
En la era de la economía basada en el conocimiento, la Gestión del Conocimiento (GC) se considera como un recurso estratégico crítico para que las empresas creen y mantengan su ventaja competitiva en entornos dinámicos (Liebeskind, 2001). Cada vez más organizaciones aceptaron la importancia de la GC, enfatizaron sus iniciativas, y realizaron vastas inversiones en el desarrollo de estrategias para dicha gestión (Roos et al., 2007). La apariencia del conocimiento como un recurso estratégico está transformando las formas de competencia y el proceso de elaboración de estrategias y
\end{abstract}


http://revistainvestigacionacademicasinfrontera.com

toma de decisiones, y como resultado, las organizaciones se esfuerzan por desarrollar y fortalecer sus sistemas y capacidades de GC (Von Krogh et al ., 2012).

El desarrollo de una efectiva estrategia de GC refleja cómo una organización valora y dirige su adquisición y utilización de conocimientos (Oluikpe, 2012). Por lo tanto, una estrategia que por medio de tecnologías de colaboración transfiera y comparta el conocimiento de manera ágil y efectiva, se convertirá en una importante ventaja competitiva para la toma de decisiones en Marketing. El estudio de Ling (2013), por ejemplo, confirma que la estrategia de GC modera la relación entre dicha estrategia y el desempeño organizacional.

Palabras clave: Estrategia de conocimiento, Transferencia de conocimiento, Ventaja competitiva, Toma de decisiones.

\section{Introducción}

Gestión del Conocimiento involucra tanto procesos, tecnologías y personas. Con éstas últimas se pretende que innoven, colaboren y tomen decisiones con eficacia en pro de las metas organizacionales. Para ello, se requiere que las actitudes y los comportamientos de las personas evolucionen para lograr nuevos niveles de colaboración, el intercambio internacional de ideas, información, conocimiento y trabajo en sí, en apoyo de una necesidad empresarial (Plessis, 2007). 
Año 10, Núm. 25 (Enero - junio 2017)

http://revistainvestigacionacademicasinfrontera.com
Revista de Investigación

Académica sin Frontera ISSN: 2007-8870

Actualmente las organizaciones empresariales estiman al conocimiento como su recurso más valioso y estratégico; el cual les permite afrontar los problemas y oportunidades; al considerar conocimiento para influir en los problemas y oportunidades y convertirlo así en su capacidad más importante dentro de la empresa (Zack, 1998). El diseñar estrategias de transferencia de conocimiento permite a la organización mejorar la competitividad y el rendimiento, esto se debe a que la adecuada gestión y aprovechamiento del conocimiento puede impulsar a una compañía a transformarse, ser adaptable, innovadora, inteligente y sostenible (kuan y Elaine, 2006).

Krogh (2001) define la estrategia de gestión del conocimiento como la aplicación de procesos de conocimientos a un dominio actual o nuevo para lograr metas estratégicas. Utilizar la tecnología como estrategia para trasferir conocimiento en cuanto a la toma de decisiones en marketing, permite integrar las diferentes fuentes empleadas en la empresa para poder analizar el conocimiento e información que se requiere. La tecnología que contribuye a la toma de decisiones es considerada como un sistema de información interactivo y adaptable basado en computadoras (que también soporta problemas de gestión estructurados) (Turban y Aronson, 2000). Al utilizar dichos sistemas los responsables de la toma de decisiones tienen la posibilidad de encontrar soluciones a problemas diversos. El intercambio de conocimientos con tecnología funciona como habilitador que tiene la intención de apoyar el conocimiento colaborativo de trabajo como las oportunidades y retos en las organizaciones 
Año 10, Núm. 25 (Enero - junio 2017)

http://revistainvestigacionacademicasinfrontera.com
Revista de Investigación

Académica sin Frontera ISSN: 2007-8870

(Abdullah, 2007). El compartir conocimiento se refiere a la difusión de información relevante dentro de una organización (Bartol, 2002). Stasser y Titus (2003) mostraron una relación positiva entre el intercambio de conocimientos, la utilización de los conocimientos existentes, y la calidad de la toma de decisiones.

La vinculación de sistemas de información con la Gestión de conocimiento ha permitido a las organizaciones tomar decisiones a través de un enfoque científico y consciente. Un sistema de información de marketing suministra información proveniente de diferentes fuentes, identificando con rapidez las tendencias, problemas y oportunidades que ocurren en el mercado; y permitiendo a la gerencia responder acertadamente y en menor tiempo a las situaciones dadas. Las tecnologías colaborativas proveen redes electrónicas que apoyan la comunicación, compartición y la colaboración a través de la organización mediante el intercambio de conocimiento e información, repositorios compartidos, foros de discusión y mensajería (Orlikowsky, 1997). La colaboración es un fuerte conductor para la generación de conocimiento, así como para su transferencia. El groupware puede mejorar la resolución de problemas y decisiones a través de la generación de alternativas, análisis, priorización y clasificación (Ruggles, 1998).

A continuación se exponen aspectos conceptuales sobre conocimiento y algunos términos relevantes para la investigación. A su vez, se describe la problemática de la organización de referencia para ubicar al lector. Posteriormente se plantea una 
Año 10, Núm. 25 (Enero - junio 2017)

http://revistainvestigacionacademicasinfrontera.com
Revista de Investigación

Académica sin Frontera ISSN: 2007-8870

estrategia que permita compartir y aprovechar el conocimiento de la empresa a través del uso de tecnologías. La posible implementación permitirá a la organización una solución adecuada, la cual se espera que conlleve a resultados y beneficios para utilizar apropiadamente el conocimiento organizacional. Finalmente se exponen las conclusiones que hacen referencia básicamente a las posibles contribuciones futuras de la estrategia.

\section{Marco teórico}

En este apartado se presentan aspectos conceptuales relacionados básicamente a: Conocimiento, Estrategia para Transferir Conocimiento y Toma de decisiones en Marketing.

Al conjunto de cogniciones y habilidades con los cuales los individuos suelen solucionar problemas, comprender tanto la teoría como la práctica, las reglas cotidianas al igual que las instrucciones para la acción, se le conoce como conocimiento. El cual es una mezcla fluida de experiencias, valores de la información del contexto y de la percepción del experto, que proporciona un marco para evaluar e incorporar nuevas experiencias e información. Se origina y se aplica en la mente de los conocedores (Davenport, 1998). A su vez Beckman (1997), define que el conocimiento es razonar sobre datos e información para garantizar la operación, resolución de problemas, procesos de toma de decisiones, y el aprendizaje en modo 
http://revistainvestigacionacademicasinfrontera.com

activo. En este sentido, el conocimiento se basa en datos e información, pero a diferencia de éstos siempre está ligado a las personas; forma parte integral de los individuos y representa las creencias de éstos acerca de las relaciones causales (Probst, 2001).

Nonaka y Tekeuchi (1995) definen al conocimiento como "un proceso humano y dinámico de justificar las creencias personales en busca de la verdad". Esta perspectiva sugiere que el conocimiento depende del contexto-"todo el conocimiento es local (Greertz, 1983). Partiendo de la premisa que el conocimiento es local, se hace énfasis en la necesidad de conocer dentro de la organización el conocimiento tácito disperso entre su personal, Ventura (1994) reconoce que éste es un activo intangible con un claro potencial para la construcción de una ventaja competitiva sostenible; ya que al ser imperceptible se requiere de un contexto compartido, en donde el lenguaje que se utilice sea el medio para crear significados comunes en el lugar de trabajo. Por ello, el conocimiento compartido permite a las personas entender y comprender el contexto de los otros, actuando de acuerdo con dicho conocimiento; favoreciendo espacios en los que se logre sintetizar este conocimiento e incorporarlo en las actividades claves organizacionales para impulsar un proceso continuo de innovación y mejora de la empresa.

El intercambio de conocimientos ha sido considerado como el componente más importante de la gestión del conocimiento y se define como la disposición de los 
http://revistainvestigacionacademicasinfrontera.com

empleados a compartir conocimientos con otros (King, 2007) así como sus acciones en las que los empleados difunden e intercambiar información relevante con otros miembros a través de la organización. Para facilitar éste intercambio de conocimiento se desarrollan estrategias organizacionales que impulsan procesos a través de los cuales las organizaciones acceden y utilizan sus conocimientos para la extracción y creación de valor que se convierten en un eje clave para la mejora del rendimiento (Hsu y Sabherwal, 2011). Las estrategias se centran principalmente en la captura, distribución y uso eficaz del conocimiento, y pueden considerarse como la directriz general para cosechar y aprovechar los conocimientos para múltiples objetivos empresariales (Yang, 2010).

Una estrategia apropiada es decisiva para el éxito de una empresa ya que establece una comprensión común sobre dónde residen los recursos de conocimiento de la organización, cómo articular las capacidades de conocimiento en la creación de valor y la forma de integrar actividades para la ejecución exitosa de la estrategia (Oluikpe, 2012).

Los elementos que componen una estrategia de GC como ventaja competitiva de negocios se muestra en la figura 1. 
http://revistainvestigacionacademicasinfrontera.com

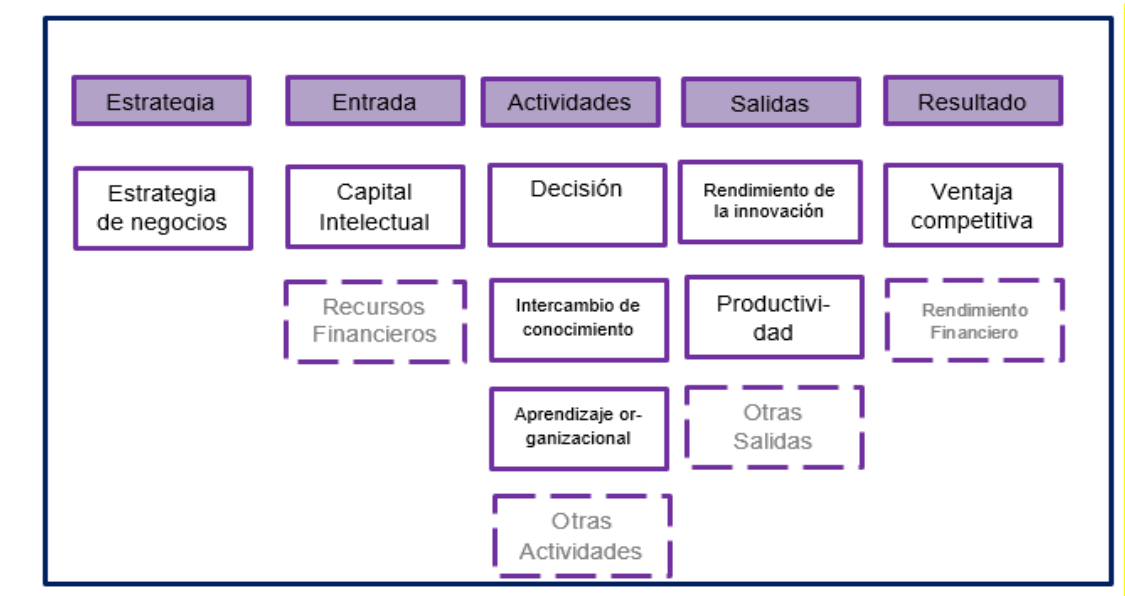

Figura 1. Elementos de una estrategia de conocimiento. Adaptado (Heisig et al., 2015)

Las estrategias a desarrollar deben cumplir con ciertas características, como es el caso de responder acertadamente cuestionamientos en relación a ¿dónde estamos ahora, dónde queremos ser, y cómo conseguimos ahí? En cuanto a la primer interrogante se hace alusión a los conocimientos que se producen, reúnen o almacenan en la empresa, es decir, aquellos productos que se crean, las formas en las cuales se gestionan los conocimientos dentro de la jerarquía institucional y qué tan efectivos son los sistemas de organización que se tienen para la GC. Un factor clave dentro de las estrategias son las metas que se definen y los tiempos que se establecen para cumplirlas, por ello, responder a ¿Dónde queremos estar? Permite a la institución centrar su atención en los cambios que la organización requiere para una estrategia 
Año 10, Núm. 25 (Enero - junio 2017)

http://revistainvestigacionacademicasinfrontera.com
Revista de Investigación

Académica sin Frontera ISSN: $2007-8870$

eficaz, así como también, delimitar aquellos mecanismos con los cuales se logre medir el valor de los esfuerzos. Por último, se encuentra el desarrollar planes de acción que describan los tres recursos: personas, procesos y tecnologías en relación a las herramientas y prácticas específicas que se utilizarán para la toma de decisiones, aquellas que sean viables para la motivación del personal en pro del progreso de la empresa (Zarinpoush et al., 2007)

Las estrategias para transferir conocimiento proporcionan una serie de ventajas a las organizaciones, una de ellas es asegurar una clara apropiación de la estrategia de transferencia de conocimiento en donde se definen las funciones clave que se desempeñarán, incluirán y afectarán en su programa de transferencia de conocimientos. A su vez, permite que las expectativas sean comunes en relación a la transferencia de conocimiento de sus ejecutivos, facilitando la selección de herramientas para trasferir conocimiento que permita que el programa sea metódico y mensurable para las necesidades empresariales identificadas. Muestra cómo la estrategia de transferencia de conocimiento se conectará a otras estrategias, ya sea en lo empresarial, en la gestión del talento, estrategia operativa, estrategia de marketing, entre otras (Trautman, 2013).

Además de lo descrito, otro aspecto positivo es que se ocupa de las actividades de trasferencia de conocimiento (como son reuniones entre experto y aprendiz), lo cual permite priorizar el trabajo en relación a las tareas diarias. Da orientación sobre los presupuestos para pagar por las actividades de transferencia de conocimientos, incluyendo la configuración inicial del programa y el mantenimiento continuo, así como 
http://revistainvestigacionacademicasinfrontera.com

los presupuestos para pagar el tiempo facturable de los expertos y de los aprendices cuando sea necesario abordarlo. Indica lo que no va a hacer para apoyar la transferencia de conocimiento, para que todos los planes operacionales se mantengan estrictos y se concentren en las tareas más importantes.

Contar con estrategias apropiadas permite a la organización la adquisición de habilidades en su fuerza laboral, aspectos que repercuten favorablemente a la productividad en la empresa. Caso contrario ocurre cuando existe ausencia de los elementos antes señalados, ya que se desarrollan diversos problemas, incluyendo (Trautman, 2013):

- Los empleados tardan demasiado en llegar a ser plenamente productivos.

- Aprenden las habilidades equivocadas o en la prioridad equivocada; posiblemente por aprender de un compañero de trabajo "experto" erróneo y adquieren malos hábitos.

- Las expectativas de la trasferencia de conocimiento no son similares entre los ejecutivos, la organización y sus socios y colaboradores, tales como proveedores externos

- Falta de conexión de los procesos de transferencia de conocimiento con otros sistemas (por ejemplo, planes operacionales, revisiones de rendimiento y compensación). 
http://revistainvestigacionacademicasinfrontera.com

\section{Modelo estratégico propuesto}

Para compartir y aprovechar el conocimiento de la organización se propone el desarrollo de una estrategia que permita tomar decisiones apropiadas para la solución de problemas, eficaces y eficientes en tiempo y forma. La estructura planteada consta de 4 fases a través de las cuales el conocimiento, así como la forma en la que se comparten y se transfiere en la organización, son clave para su progreso (figura 2). Se pretende que el modelo estratégico pueda ser replicable a cualquier entorno organizacional que permita dar solución a problemáticas similares, puesto que los elementos como personas, procesos y tecnologías, son el común denominador entre las empresas. 
http://revistainvestigacionacademicasinfrontera.com

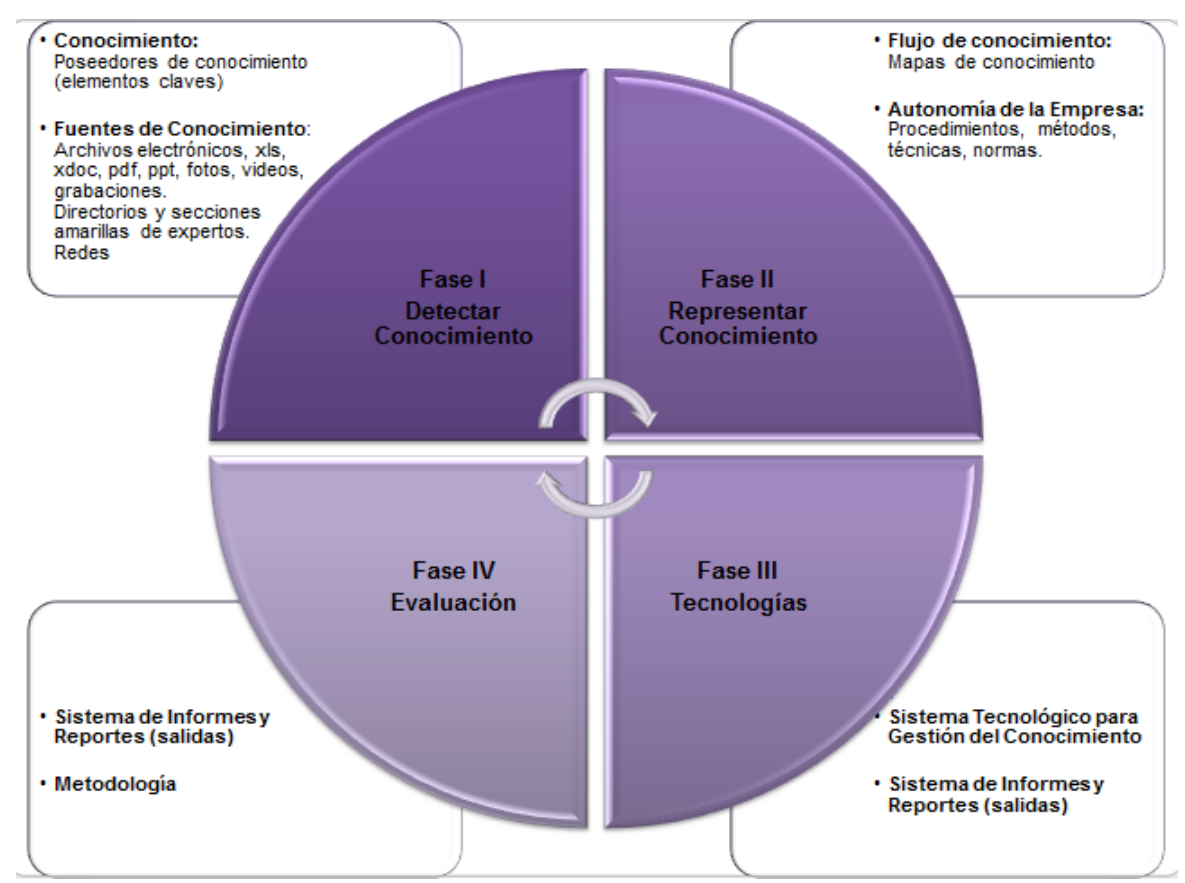

Figura 2. Estrategia Metodológica para transferir conocimiento y apoyar la toma de decisiones.

En la primera fase, la estrategia parte de la detección del conocimiento. Para su análisis, se deben recopilar todos los datos e información pertinentes, es por ello que, se seleccionarán aquellas herramientas cualitativas apropiadas como es el caso de entrevistas, encuestas, cuestionarios, entre otras, que permitan tener la mejor comprensión posible del entorno de la empresa. A su vez hay que determinar las fuentes de consulta o procedencia del conocimiento y registrarlo para trazar estrategias futuras. El conocimiento es inherente a la persona por tanto es complicado ubicarlo fácilmente; por ello al realizar la identificación del conocimiento se han de 
Año 10, Núm. 25 (Enero - junio 2017)

http://revistainvestigacionacademicasinfrontera.com
Revista de Investigación

Académica sin Frontera ISSN: 2007-8870

considerar los vacíos de cada trabajador, su rendimiento y las capacidades de distribuir lo que saben y poder encontrar las lagunas de conocimiento existente en la organización.

Una vez que se aplican las herramientas de recolección de datos, en la siguiente fase, se debe de representar el conocimiento para trazar estrategias de transferencia y lograr el éxito organizacional. Al utilizar herramientas como lo son mapas de conocimiento se contribuye a ubicar donde se encuentra el conocimiento en la organización (Pietrzak, 2015). Los mapas de las fuentes del conocimiento muestran qué personas de una organización, de un equipo de trabajo o del entorno contribuyen con sus conocimientos a las actividades y procesos de la organización (Probst, 2001). Posteriormente, en la tercera etapa de la metodología se hace uso de las tecnologías, las cuales se refieren a toda herramienta que se utiliza para la solución de problemas, toma de decisiones o satisfacer necesidades de la organización. En esta etapa se incluye procesos, habilidades, técnicas, métodos, etc. para el logro de objetivos. Una ventaja competitiva es utilizar el conocimiento, los recursos y tecnologías para su gestión, con los cuales existiría una adecuada transferencia entre las personas. Por ello, es necesario que el conocimiento sea almacenado en medios electrónicos para facilitar su explotación y utilización.

En la última fase, se debe llevar a cabo la evaluación, donde se espera que ésta tecnología permita a los responsables de tomar las decisiones sobre estrategias de venta y nuevos negocios, trabajen simultáneamente y compartan conocimiento e información. La autoevaluación deberá mostrar qué áreas mejoraron y qué aspectos 
http://revistainvestigacionacademicasinfrontera.com

hay que seguir modificando para poder tener un esquema que favorezca el crecimiento y desarrollo empresarial.

\section{Resultados y beneficios}

Se pretende que el uso de la tecnología para transferir conocimiento como estrategia se traduzca en el logro de los objetivos organizacionales, así como un mejor rendimiento y competitividad en el mercado, siendo ésta última su punto más fuerte en marketing.

Con la aplicación de herramientas apropiadas dentro de la compañía, se pretende que el trabajar en colaboración genere mayores beneficios que el hacerlo de forma individual. El software para el trabajo en grupo propiciará el compartir conocimiento adecuado en donde el rendimiento se verá favorecido en relación a la toma de decisiones; trayendo consigo que la diferentes personas puedan trabajar compartiendo información y conocimiento para el desarrollo y culminación de los proyectos que se realizan dentro de la institución. 
Año 10, Núm. 25 (Enero - junio 2017)

http://revistainvestigacionacademicasinfrontera.com
Revista de Investigación

Académica sin Frontera ISSN: 2007-8870

\section{Conclusiones}

De acuerdo a lo revisado en la literatura y analizando casos de éxito de empresas en donde se han implementado tecnologías para compartir conocimiento, así como también los procesos y resultados que se llevaron a cabo en la implementación de la estrategia utilizada en la organización se puede concluir que la estructura de la industria se ve favorecida al utilizar dentro de su sistema aquellas herramientas apropiadas de groupware ya que potencia el proceso de toma de decisiones al ser éstas efectivas y eficaces a la hora de la resolución de la diversidad de problemáticas que se presentan.

El contar con un sistema que favorezca la toma de decisiones se espera que tenga un impacto sustancial en la empresa, traduciéndose a nuevas oportunidades de negocios y estrategias de venta que en la actualidad requieren de un nivel de competencia elevado para poder colocarse en el mercado de forma exitosa. Cabe señalar que los procesos en los que se involucra la toma de decisiones deben estar sometidos a revisiones y autoevaluaciones que le permitan al sistema mantenerse actualizado para lograr efectividad en su función.

\section{Argumentación de la propuesta de solución}

Las organizaciones empresariales actualmente manifiestan una situación crítica en relación a la forma en que toman las decisiones de manera efectiva y acertada. Las cuales están asociadas a los cambios en los mercados, la implementación de diversos 
Año 10, Núm. 25 (Enero - junio 2017)

http://revistainvestigacionacademicasinfrontera.com
Revista de Investigación

Académica sin Frontera ISSN: 2007-8870

productos, la preferencia del consumidor, entre otros. Lo antes descrito, se convierte en uno de los principales retos para las empresas, ya que, las organizaciones pueden poseer recursos materiales similares entre ellas; generando pérdidas y un impacto negativo en el mercado. Por esta razón, tanto el conocimiento y su gestión se convierten en elementos claves para asegurar el éxito de la organización. Uno de los aspectos sustanciales del conocimiento es su trasferencia, la cual permite a los empleados compartir a través de distintas estrategias y métodos los datos que se requieren para la toma de decisiones.

La era digital aporta múltiples posibilidades con las que se puede mejorar la gestión y transferencia de conocimiento. Precisamente se deben considerar las posibilidades que aportan diferentes tecnologías y herramientas. Sin embargo, para aprovechar realmente el impulso creado por las nuevas tecnologías, es importante encuadrar estas acciones de gestión y transferencia como ejes estratégicos de las organizaciones frente a la competencia.

Para lograr una adecuada trasferencia del conocimiento Rodríguez (2009) sugiere que organización utilice herramientas que faciliten la captura, conservación, organización y procesamiento, y sobre todo, la difusión del conocimiento de tal forma que se transforme dicho recurso que se encontraba disperso en una solución eficaz. La transferencia de conocimiento tiene la oportunidad de hacer que una compañía sea más atractiva para los trabajadores con talento, es decir, al establecer una estrategia 
Año 10, Núm. 25 (Enero - junio 2017)

http://revistainvestigacionacademicasinfrontera.com
Revista de Investigación

Académica sin Frontera ISSN: 2007-8870

que genere un programa estructurado de transferencia de conocimiento, los tomadores de decisiones pueden mostrar cómo la organización invertirá en su crecimiento de habilidades y acelerará su incorporación, traduciéndose en un crecimiento empresarial, es decir, se generen nuevos servicios o productos innovadores para alcanzar metas y objetivos establecidos, permitiendo un desarrollo sostenible.

Según Trautman (2013) las organizaciones deben desarrollar una estrategia de transferencia de conocimiento puesto que adquirir, compartir y mantener el conocimiento es fundamental para el desempeño. Los sistemas de transferencia de conocimientos, tanto formales como informales, ya son la forma en que las personas aprenden sus trabajos. Numerosos estudios independientes muestran que entre el $70 \%$ y el $80 \%$ de la adquisición de conocimientos ocurre en el trabajo independientemente de cuánta educación formal haya estado disponible. En ausencia de una estrategia y un plan táctico, la adquisición de habilidades de su fuerza laboral se enfrentan a una variedad de problemas.

Las ideas y herramientas propuestas en el presente documento sirven de igual manera hacia la gestión-transferencia del conocimiento en distintas organizaciones o empresas para obtener valor y riqueza del conocimiento interno a través de su capacidad para transferirlo de forma eficiente. Lo anterior se traduce en mejores 
Año 10, Núm. 25 (Enero - junio 2017)

http://revistainvestigacionacademicasinfrontera.com
Revista de Investigación Académica sin Frontera ISSN: 2007-8870

estrategias de negocios, nuevos clientes y mercados, nuevas y mejores formas de marketing y sobre todo una ventaja competitiva frente a sus competidores.

\section{Referencias}

1. Abdullah, R. y Mohd Hasan, S. (2007). Facilitating Knowledge Sharing With Groupware among Faculty Communities in Higher Learning Institution

2. Bartol, K. M., y Srivastava, A. (2002). Encouraging knowledge sharing: The role of organizational reward systems. Journal of Leadership \& Organizational Studies, 9, 64-76.

3. Beckman, T. (1997). A Metodology of Knowledge Management. In Proceedings of thelnternational Association of Science and Technology for Development (IASTED) Al and Soft Computing Conference. Banff, Canada.

4. Davenport, T.H., Prusak L. 1998. Working Knowledge: How Organizations Manage What They Know, Boston: Harvard Business School Press.

5. Greertz, C (1983). Local Knowledge, Further Essays in Interpretative Anthropology, Nueva York, Basic Books

6. Heisig, P. (2015), "Future research about knowledge and knowledge management - results from the global knowledge research network study", in Bolisani, E. and Handzic, M. (Eds), Advances In Knowledge Management Celebrating Twenty Years of Research and Practice, Springer, Berlin, London, NY.

7. Heisig, P. (2014), "Knowledge management - advancements and future research needs - results from the global knowledge research network study", British Academy of Management (BAM) Conference, Belfast.

8. King, W. 2007. A research agenda for the relationships between culture and knowledge management, Knowledge and Process Management, 14 (3), pp. 226-236.

9. Kuan Y. Wonga and, Elaine A. (2006), Development of a knowledge management initiative and system: A case study, ELSEVIER, Expertm Systems with Applications 30 (2006) 633-641. 


\section{http://revistainvestigacionacademicasinfrontera.com}

10. Liebeskind, J.P. (2001), "Managing intellectual capital: organizational, strategic and policy dimensions", Academy of Management Review, Vol. 26 No. 4, pp. 664-665.

11. Ling, Y.H. (2013), "The influence of intellectual capital on organizational performance-knowledge management as moderator", Asia Pacific Journal of Management, Vol. 30 No. 3, pp. 937-964.

12. Orlikowsky, W., y Hofman, D. (1997). An improvisational model of change management: The case of groupware technologies.

13. Oluikpe, P. (2012), "Developing a corporate knowledge management strategy", Journal of Knowledge Management, Vol. 16 No. 6, pp. 862-878.

14. Pietrzak, Michal, Joanna Paliszkiewicz, Krzysztof Jalosinski, and Andrzej Brzozowski. (2015). "A case study of strategic group map application used as a tool for knowledge management." Journal Of Computer Information Systems 55, no. 2: 68-77.

15. Plessis, M. (2007), "The role of knowledge management in innovation", Journal of Knowledge Management, Vol. 11 No. 4, pp. 20-29.

16. Probst, G., Raub, S. y Romhardt, K. (2001). Administre el conocimiento.México:Prentice Hall.

17. Roos, G., Roos, G., Pike, S. and Fernstrom, L. (2007), Managing Intellectual Capital in Practice.

18. Turban, E., and J. Aronson. 2000. Decision Support Systems and Intelligent Systems, 6th edn. Upper Saddle River, NJ: Prentice-Hall.

19. Trautman, S. (2013) Teach What Your Know: A Practical Leader's Guide to Knowledge Transfer Using Peer Mentoring, Steve Trautman. Prentice Hall, 2007.

20. Trautman, S. (2012) The Executive Guide to High-Impact Talent Management, David DeLong and Steve Trautman. McGrawHill, 2011.

21. Ventura, Victoria, J. (1994): Análisis competitivo de la empresa. Civitas.Madrid.

22. Von Krogh, G., Nonaka, I. and Rechsteiner, L. (2012), "Leadership in organizational knowledge creation: a review and framework", Journal of Management Studies, Vol. 49 No. 1, pp. 240-277.

23. Stasser, G., y Titus, W. (2003). Hidden profiles: A brief history. Psychological Inquiry, 14, 304-313. 
Año 10, Núm. 25 (Enero - junio 2017)

Revista de Investigación

Académica sin Frontera

ISSN: 2007-8870

http://revistainvestigacionacademicasinfrontera.com

24. Zack, M. (1998) "What Knowledge-Problems Can Information Technology Help to Solve," in Pro-ceedings of the Fourth Americas Conference on Information Systems, E. Hoadley and I. Ben-Basat (eds.), Baltimore, MD, August, pp. 644646. 